\title{
Coexistencia de linfoma plasmablástico, sarcoma de Kaposi y enfermedad de Castleman en un paciente con infección por virus de inmunodeficiencia humana
}

\author{
Alejandro Avilés-Salas, Patricia Cornejo-Juárez y Pedro de J. Sobrevilla-Calvo
}

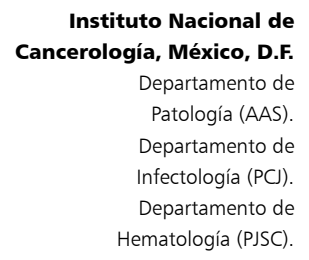

Recibido: 8 de enero de 2010 Aceptado: 20 de noviembre de

2010

Correspondencia a: Alejandro Avilés Salas alejandroaviles2001@yahoo.com

\section{Coexistence of plasmablastic lymphoma, Kaposi sarcoma and Castleman disease in a patient with HIV infection}

Plasmablastic lymphoma (PL) is an uncommon B-cell lymphoma that is strongly associated with human immunodeficiency virus (HIV) infection, and displays distinctive affinity for extranodal presentation in the oral cavity. We report the case of a PL involving the stomach in a 36 year-old man HIV+ patient, associated with Kaposi sarcoma (KS) in sections adjacent to lymphoma. He had a positive history of Castleman disease and KS in a lymphoid node biopsy.

Key words: Plasmablastic lymphoma, Castleman disease, Kaposi sarcoma, HIV.

Palabras clave: Linfoma plasmablástico, enfermedad de Castleman, sarcoma de Kaposi, VIH.

\section{Introducción}

A partir de 1981 con la emergencia del SIDA se ha observado una estrecha asociación entre la infección por virus de inmunodeficiencia humana (VIH) y el desarrollo de diferentes neoplasias, las cuales incluyen: sarcoma de Kaposi (SK), linfoma no Hodgkin (LNH), cáncer del canal anal y cáncer de cérvix.

Los linfomas afectan a $5-10 \%$ de los pacientes infectados por VIH, siendo la segunda neoplasia más común en esta población después del SK. El riesgo de desarrollar un $\mathrm{LNH}$ es 150 a 250 veces mayor en pacientes con infección por VIH que en la población general ${ }^{1}$. Los linfomas observados son predominantemente linfomas de estirpe B: linfoma difuso de células grandes (LDCG), linfoma de Burkitt (LB), linfoma primario de serosas (LPS) y linfoma plasmablástico (LP) ${ }^{2}$.

El virus herpes (tipo 8) asociado a sarcoma de Kaposi (VHSK) se ha relacionado con la patogénesis del SK, del LPS y de la enfermedad de Castleman difusa de células plasmáticas (ECDCP) $)^{3,4}$; por otro lado, el virus de Epstein Barr (VEB) se ha relacionado como factor etiológico del LP y del LDCG 5 .

El LP tiene una fuerte predilección por la cavidad oral $^{6,7}$; sin embargo, también se ha informado en otras localizaciones incluyendo: estómago, pulmón, ganglios linfáticos, región anorectal y senos paranasales ${ }^{8-10}$.

\section{Caso Clínico}

Hombre de 36 años de edad, soltero, procedente de medio socioeconómico bajo, quien inició su padecimiento en agosto de 2005 con aumento progresivo de volumen de las cadenas ganglionares cervicales, submaxilares, supraclaviculares e inguinales. Posteriormente, en enero de 2006 presentó diarrea acompañada de náuseas, vómitos, sensación de plenitud abdominal, fiebre y pérdida de $5 \mathrm{~kg}$ de peso, motivo por el cual acudió al Hospital General de México donde se le realizó una biopsia de un ganglio linfático cervical que se diagnosticó como ECDCP co-existente con SK. Además se le realizó prueba de ELISA para VIH que resultó positiva. Posteriormente, el paciente fue referido al Instituto Nacional de Cancerología para su valoración y manejo. A su ingreso se encontraba orientado, con taquicardia, febril, y con índice Karnofsky de $80 \%$. En la exploración física se palparon múltiples adenopatías de 1,5 a $3 \mathrm{~cm}$ de diámetro en varias cadenas ganglionares, sin compromiso de mediastino, hígado ni bazo y no se informaron lesiones cutáneas de SK.

En la revisión de los nuevos cortes del bloque de parafina del ganglio linfático se observó pérdida de la arquitectura normal a expensas de áreas de bordes mal definidos, constituidas por células fusiformes dispuestas en fascículos, con formación de grietas vasculares y presencia de pigmento hemático y macrófagos (Figura 1a). 
En el resto del ganglio linfático se apreció expansión del espacio interfolicular y medular por células plasmáticas, de aspecto maduro. Se realizó estudio de inmunohistoquímica el cual resultó positivo en las células plasmáticas para CD138, kappa y lambda, lo cual corroboró el diagnóstico de ECDCP y SK (Figura 1b).

Los datos relevantes de laboratorio fueron: leucocitos: $11.900 / \mathrm{mm}^{3}$, neutrófilos absolutos: $8.500 / \mathrm{mm}^{3}$, plaquetas:

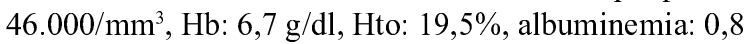
g/dl y DHL: 428 UI/1. La carga viral fue de 57.800 copias ARN/ml, CD4: 29 céls $/ \mathrm{mm}^{3}$ y CD8: 490 céls $/ \mathrm{mm}^{3}$, lo que corroboró un estado de infección C3. Durante su evolución se exacerbó la náusea y el vómito, provocándole deshidratación, desequilibrio hidro-electrolítico e insuficiencia renal aguda. Se le efectuó endoscopia y biopsia de estómago con la sospecha diagnóstica de SK. En el estudio histopatológico, en algunos fragmentos se observó neoplasia vascular con características microscópicas similares a las descritas en la biopsia de ganglio linfático, consistentes con SK. Los fragmentos restantes estaban infiltrados por neoplasia maligna de crecimiento difuso con patrón en cielo estrellado (Figura 2a). Las células eran grandes, tenían núcleos ovoides, nucléolo prominente y abundante citoplasma, lo cual les confería un aspecto plasmablástico (Figura 2b). Se realizó estudio de inmunohistoquímica (Tabla 1), siendo las células neoplásicas positivas para CD138 y cadenas ligeras lambda (Figura 2c) y negativas para CD20 y proteína latente de membrana (LMP-1). Basados en el aspecto morfológico y el resultado de inmunohistoquímica se concluyó el diagnóstico de mucosa gástrica infiltrada por LP co-existente con SK.

Se inició tratamiento con ciclofosfamida, doxorrubicina, vincristina y prednisona (CHOP); sin embargo, el paciente desarrolló falla orgánica múltiple motivo por el cual ingresó a la unidad de cuidados intensivos. A petición de su familiar el paciente fue egresado por alta voluntaria, a pesar de las condiciones de salud y el pronóstico grave a corto plazo.

\section{Discusión}

A partir de 1996, con el uso de la terapia anti-retroviral de gran actividad (TARGA) se ha registrado una reducción dramática en la morbilidad y mortalidad relacionada al SIDA; sin embargo, no se ha observado un efecto importante en la incidencia de $\mathrm{LNH}^{1,11}$.

El LP representa aproximadamente 2,6\% de todos los $\mathrm{LNH}$ asociados a infección por $\mathrm{VIH}^{12}$. En la patogénesis de los linfomas asociados a VIH los virus juegan un papel importante, así como alteraciones en c-myc, p53 y Bcl- $6^{13}$. La infección por EBV es común en el LDCG, LB y LP ${ }^{14}$, por otro lado el VHSK se ha implicado en la patogénesis del LP, el LPS y la ECDCP ${ }^{15,16}$. De los casos informados de LP, aproximadamente $76 \%$ se han asociado a EBV y entre 17 y $38 \%$ a VHSK. El papel etiológico del VHSK ha sido cuestionado debido a la incapacidad de reproducir estos resultados de forma consistente ${ }^{6,17}$. Co-factores adicionales, tales como hormonas, predisposición genética y/o co-infección con otros agentes infecciosos, al parecer, son necesarios dentro del proceso neoplásico.

El SK es la neoplasia más común en pacientes infectados con VIH y es la forma más agresiva y con mayor resistencia al tratamiento. Su incidencia se ha reducido considerablemente en los últimos años como resultado del advenimiento de la TARGA, e incluso, en algunos casos, puede remitir completamente.

La ECDCP es un trastorno linfoproliferativo caracterizado por linfadenopatía, fiebre e hipergamaglobulinemia; así como mantos de células plasmáticas en el espacio inter-folicular ${ }^{18}$. Los pacientes frecuentemente muestran anormalidades inmunológicas y desarrollan tumores secundarios como SK, linfoma de Hodgkin, plasmocitomas y $\mathrm{LNH}^{15}$. Aun cuando la mayoría de las lesiones son de origen policlonal y se consideran reactivas, se ha informado que, bajo ciertas circunstancias, los pacientes con ECDCP pueden progresar hacia un LNH agresivo, siendo el LP e inmunoblástico los tipos más frecuentemente descritos ${ }^{19}$.
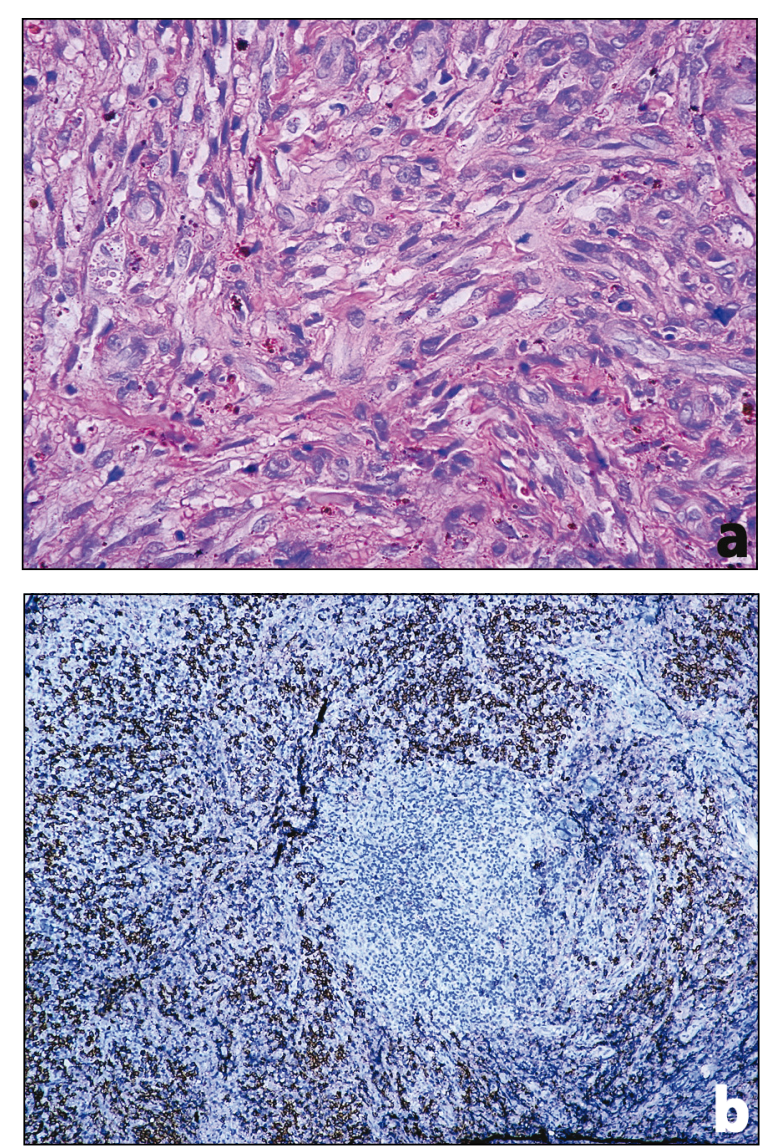

Figura 1. (a) Biopsia de ganglio linfático con áreas de SK. Se observa lesión con patrón de crecimiento sólido, constituida por células fusiformes con formación de grietas vasculares. Hay depósito de hemosiderina (Hematoxilina-eosina, 400X). (b) Áreas de ECDCP. El espacio inter-folicular muestra mantos de células plasmáticas maduras, mezcladas con linfocitos y células de aspecto monocitoide. Las células plasmáticas son positivas a CD 138 (Técnica de inmunohistoquímica, 200X). 
Figura 2. (a) Áreas de mucosa gástrica con LP. La neoplasia infiltra y sustituye la mucosa gástrica y muestra patrón en cielo estrellado (Hematoxilina-eosina, 200X). (b) Las células tienen núcleos grandes, cromatina fina, nucléolos prominentes, y el citoplasma es amplio y basófilo (plasmablástico). Se identifican numerosas mitosis (hematoxilina-eosina, 400X) (c) Las células neoplásicas son intensamente positivas a cadenas ligeras lambda (Técnica de inmunohistoquímica, 400X).

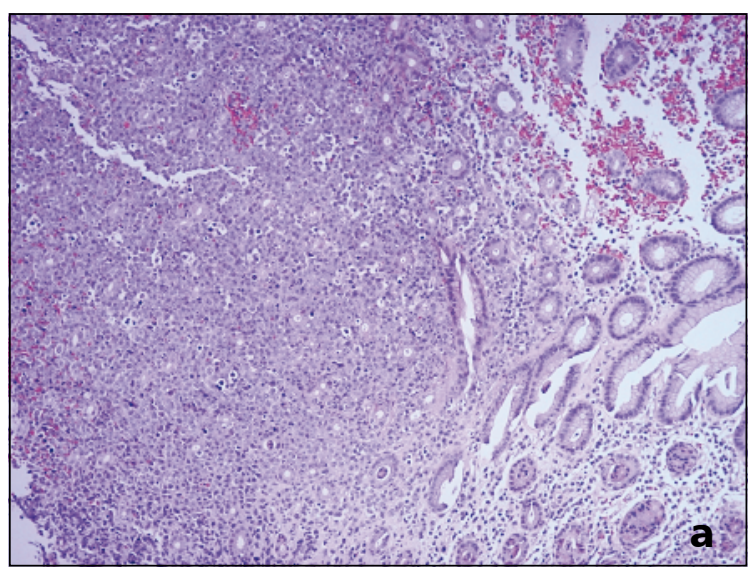

\begin{tabular}{|cc|}
\hline Tabla 1. Estudio de inmunohistoquímica \\
\hline Anticuerpo & Resultado \\
\hline CD 138 & + \\
\hline Lambda & + \\
\hline Kappa & - \\
\hline LMP-1 & - \\
\hline CD 20 & - \\
\hline CD $79 a$ & - \\
CD 45 Ro & - \\
\hline CD 3 & - \\
\hline CD 30 & - \\
\hline
\end{tabular}
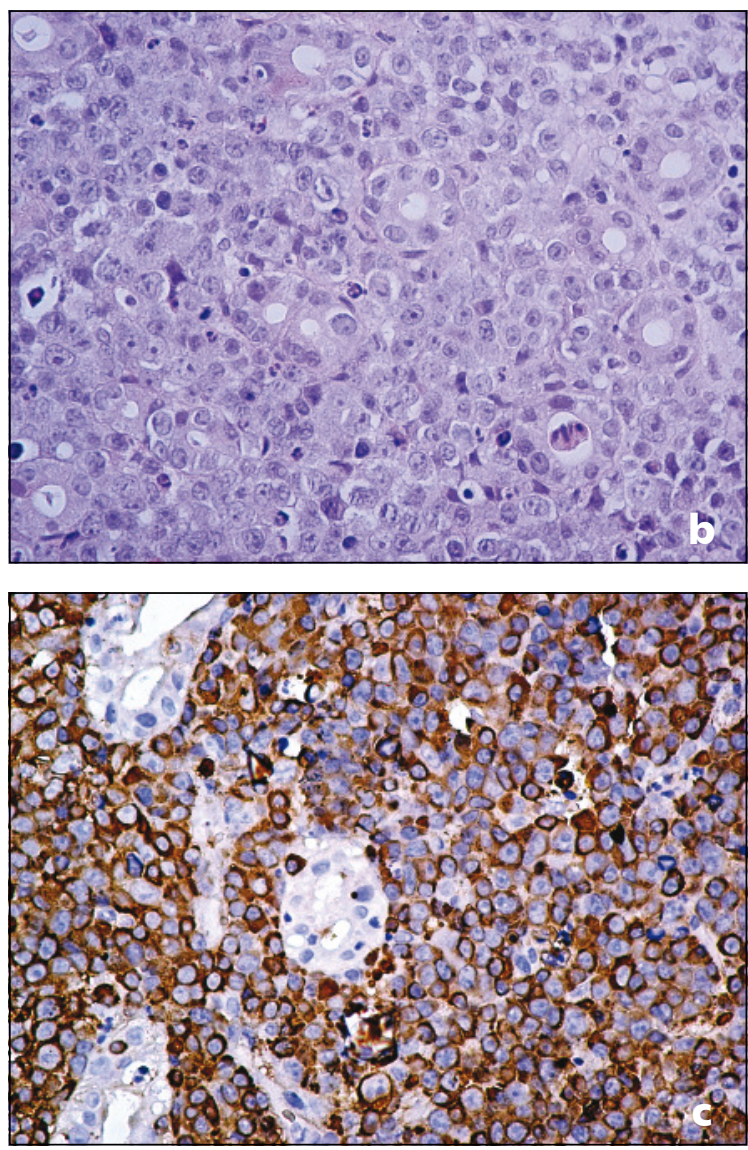

Los pacientes con infección por VIH y LNH frecuentemente tienen enfermedad avanzada y/o extra-ganglionar y síntomas B en aproximadamente $80 \%$ de los casos. El sitio más común de afección extra-ganglionar es el tracto gastrointestinal, particularmente el intestino delgado, el estómago y la región perianal. El compromiso hepático se ha informado en una cuarta parte de los pacientes, la infiltración de la médula ósea en $20 \%$ y la afección leptomeníngea puede estar presente al momento del diagnóstico y ser asintomática en más de $20 \%$ de los casos $^{11}$.

Deloose y cols, informaron infección por VHSK en $38 \%$ de los pacientes infectados por VIH con linfomas inmunoblásticos/plasmablásticos. Estos linfomas frecuentemente están precedidos por SK y predominantemente afectan el tracto gastrointestinal ${ }^{4,5}$. En el caso informado, el paciente mostró al inicio de su padecimiento la coexistencia de SK y ECDCP a nivel ganglionar y, poco tiempo después, la co-existencia de LP y SK en estómago.

Las células del LP se caracterizan por tener citoplasma basófilo, núcleo excéntrico y nucléolo central prominente. Tienen crecimiento cohesivo, son relativamente uniformes y frecuentemente muestran patrón en cielo estrellado ${ }^{20}$. Colomo y cols, describieron dos subgrupos de LP; el primero de tipo mucosa oral, constituido por una población monomórfica de inmunoblastos, la gran mayoría de los se presentó en la cavidad oral y sólo $13 \%$ en ganglios linfáticos. El segundo subgrupo con diferenciación plasmacítica, compuesto por inmunoblastos, plasmablastos pero con diferenciación a célula plasmática madura; sólo $33 \%$ de los pacientes tenían infección por VIH; el EBV fue detectado en $64 \%$ de los casos y $44 \%$ se presentaron en ganglios linfáticos ${ }^{21}$. Por definición son CD138, EMA e Ig citoplásmica positivos y CD20 negativos. La detección de EBV por inmunohistoquímica utilizando LMP-1 es muy baja; por el contrario, al utilizar hibridación in situ con EBER el porcentaje aumenta hasta $76 \% \%^{5,6,21}$. En el presente caso el diagnóstico de LP se sustentó en base al aspecto morfológico y al perfil de inmunohistoquímica, aun cuando no se documentó la asociación con EBV.

En general, los linfomas asociados a infección por VIH tienen un comportamiento biológico agresivo y rápidamente fatal ${ }^{1,5,22}$; sin embargo, el diagnóstico temprano y el tratamiento adecuado para ambas condiciones han mejorado significativamente el pronóstico de estos pacientes. En el caso informado, la terapia anti-retroviral pacientes eran $\mathrm{VIH}+/ \mathrm{EBV}+$, aproximadamente la mitad 
que debió haber recibido es la indicada por el Centro Nacional para la Prevención y Control del VIH/SIDA en México, la que consiste de efavirenz $600 \mathrm{mg}, 1$ tableta cada 24 horas por la noche, más Truvada ${ }^{\circledR}$ (emtricitabina $200 \mathrm{mg} /$ tenofovir $300 \mathrm{mg}$ ) 1 tableta cada 24 horas, o como régimen alternativo Kaletra ${ }^{\mathbb{B}}$ (lopinavir $200 \mathrm{mg} /$ ritonavir $100 \mathrm{mg}) 2$ tabletas cada 12 horas, más Truvada ${ }^{\circledR} 1$ tableta cada 24 horas.

Por otro lado, aun cuando en nuestro caso se propuso aplicar CHOP, existen otros esquemas de quimioterapia utilizados en estos pacientes tales como: $\mathrm{EPOCH}$ (etopósido, doxorrubicina, vincristina, ciclofosfamida y prednisona), CODOX-M/IVAC (ciclofosfamida, vincristina, doxorrubicina, metotrexate, ifosfamida, etopósido y citarabina), ACVBP (doxorrubicina, ciclofosfamida, vindesina, bleomicina y prednisona) e hiper-CVAD (ciclofosfamida, metotrexate, citarabina, vincristina, doxorrubicina y dexametasona) $)^{17,23}$. Con la finalidad de reducir la toxicidad hematológica se ha propuesto el esquema de EPOCH a dosis ajustada, utilizando: etopósido $\left(50 \mathrm{mg} / \mathrm{m}^{2} /\right.$ día), doxorrubicina $\left(10 \mathrm{mg} / \mathrm{m}^{2} /\right.$ día $)$, vincristina $(0,4 \mathrm{mg} /$ $\mathrm{m}^{2} /$ día) en infusión continua y prednisona $\left(60 \mathrm{mg} / \mathrm{m}^{2} /\right.$ día $)$ vía oral; así como ciclofosfamida, ajustando su dosis en relación a la cuenta de células CD4+; mayor de 100/ $\mathrm{mm}^{3}$ a dosis de $375 \mathrm{mg} / \mathrm{m}^{2} /$ día y menor de $100 / \mathrm{mm}^{3}$ a dosis de $187 \mathrm{mg} / \mathrm{m}^{2} /$ día, el primer ciclo. Posteriormente, la dosis de ciclofosfamida se ajusta por arriba o bajo de $187 \mathrm{mg} /$ $\mathrm{m}^{2}$, en relación a la cuenta absoluta de neutrófilos, con una dosis máxima de $750 \mathrm{mg} / \mathrm{m}^{2} 24$.

El diagnóstico diferencial debe incluir linfoma inmu- noblástico, LPS, LB con diferenciación plasmocitoide y mieloma poco diferenciado ${ }^{14}$; sin embargo, es importante tener en cuenta otros diagnósticos no hematológicos como carcinoma poco diferenciado y melanoma 5 .

Finalmente, factores que han sido asociados a mal pronóstico en esta población incluyen: edad $>35$ años, cuenta de CD4 $<100$ céls $/ \mathrm{mm}^{3}$ al momento del diagnóstico, pobre estado funcional, infiltración de la médula ósea, DHL sérica elevada, estadio III o IV, y pobre respuesta a TARGA ${ }^{1,11}$. En la práctica clínica, el diagnóstico temprano de linfomas asociados a VIH, así como la capacidad de inhibir el crecimiento tumoral y la replicación viral son de utilidad pronóstica. En el futuro, la comprensión de la patogénesis y la división en subgrupos serán la base de las estrategias terapéuticas dirigidas a este tipo de lesiones.

\section{Resumen}

El linfoma plasmablástico (LP) es un linfoma de células $\mathrm{B}$ poco común que está fuertemente asociado con la infección por el virus de inmunodeficiencia humana (VIH), y muestra una afinidad característica de presentación extra-ganglionar en la cavidad oral. Informamos el caso de un LP afectando el estómago en un paciente masculino de 36 años de edad con infección por VIH, asociado con sarcoma de Kaposi (SK) en áreas adyacentes al linfoma. Tenía el antecedente de enfermedad de Castleman y SK en una biopsia de ganglio linfático.

\section{Referencias}

1.- Cornejo-Juárez P, Volkow-Fernández P, Avilés-Salas A, Calderón-Flores E. AIDS and non-Hodgkin's lymphoma. Experience at an oncological center in México. Rev Invest Clin 2008; 60: 375-81.

2.- Folk G S, Abbondanzo S L, Childers E L, Foss R D. Plasmablastic lymphoma: a clinicopathologic correlation. Ann Diagn Pathol 2006; 10: 8-12.

3.- Parravicini C, Chandran B, Corbellino M, Berti E, Paulli M, Moore P S, et al. Differential viral protein expression in Kaposi's sarcomaassociated herpesvirus-infected diseases: Kaposi's sarcoma, primary effusion lymphoma, and multicentric Castleman's disease. Am J Pathol 2000; 156: 743-9.

4.- Deloose S T, Smit L A, Pals F T, Kersten M J, van Noesel C J, Pals S T. High incidence of Kaposi sarcoma-associated herpesvirus infection in VIH-related solid immunoblastic/ plasmablastic diffuse large B-cell lymphoma. Leukemia 2005; 19: 851-5.

5.- Rafaniello Raviele P, Pruneri G, Maiorano E.
Plasmablastic lymphoma: a review. Oral Dis 2009; 15: 38-45

6.- Delecluse H J, Anagnostopoulos I, Dallenbach F, Hummel M, Marafioti T, Schneider U, et al. Plasmablastic lymphomas of the oral cavity: a new entity associated with the human immunodeficiency virus infection. Blood 1997; 89: 1413-20.

7.- Borrero J J, Pujol E, Pérez S, Merino D, Montaño A, Rodríguez F J. Plasmablastic lymphoma of the oral cavity and jaws. AIDS 2002; 16: 1979-80.

8.- Chetty R, Hlatswayo N, Muc R, Sabaratnam R, Gatter K. Plasmablastic lymphoma in $\mathrm{HIV+}$ patients: an expanding spectrum. Histopathology 2003; 42: 605-9.

9.- Pruneri G, Graziadei G, Ermellino L, Baldini L, Neri A, Buffa R. Plasmablastic lymphoma of the stomach. A case report. Haematologica 1998; 83: 87-9.

10.- Lin Y, Rodrigues G D, Turner J F, Vasef M A. Plasmablastic lymphoma of the lung: report of a unique case and review of the literature. Arch Pathol Lab Med 2001; 125: 282-5.

11.- Powles T, Matthews G, Bower M. AIDS related systemic non-Hodgkin's lymphoma. Sex Transm Infect 2000; 76: 335-41.

12.- Carbone A. AIDS-relate non-Hodgkin's lymphomas: from pathology and molecular pathogenesis to treatment. Hum Pathol 2002; 33: 392-404.

13.- Carbone A. Emerging pathways in the development of AIDS-related lymphomas. Lancet Oncol 2003; 4: 22-9.

14.- Dong H Y, Scadden D T, de Leval L, Tang Z, Isaacson P G, Harris N L. Plasmablastic lymphoma in VIH-positive patients: an aggressive Epstein-Barr virus-associated extramedullary plasmacytic neoplasm. Am J Surg Pathol 2005; 29: 1633-41.

15.- Cesarman E, Knowles D M. Kaposi's sarcomaassociated herpesvirus: a lymphotropic human herpesvirus associated with Kaposi's sarcoma, primary effusion lymphoma, and multicentric Castleman's disease. Semin Diagn Pathol 1997; 14: 54-66.

16.- Oksenhendler E, Boulanger E, Galicier L, Du M Q, Dupin N, Diss T C, et al. High incidence of Kaposi sarcoma-associated herpesvirus-related non-Hodgkin lymphoma 
in patients with HIV infection and multicentric Castleman disease. Blood 2002; 99: 2331-6.

17.- Riedel D J, González-Cuyar L F, Zhao X F, Redfield R R, Gilliam B L. Plasmablastic lymphoma of the oral cavity: a rapidly progressive lymphoma associated with HIV infection. Lancet Infect Dis 2008; 8: 261-7.

18.- Sobrevilla-Calvo P de J, Avilés-Salas A, Cortés-Padilla D E, Rivas-Vera S.

Características clínico-patológicas de la enfermedad de Castleman. Experiencia en el Instituto Nacional de Cancerología. Cir Cir 2009; 77: 187-92.

19.- Dupin N, Diss T L, Kellam P, Tulliez M, Du M Q, Sicard D, et al. HHV-8 is associated with a plasmablastic variant of Castleman disease that is linked to HHV-8 positive plamablastic lymphoma. Blood. 2000; 95: 1406-12.

20.- Gujral S, Shet T M, Kane S V. Morphological spectrum of AIDS-related plasmablastic lymphomas. Indian J Pathol Microbiol 2008; 51 : 121-4.

21.- Colomo L, Loong F, Rives S, Pittaluga S, Martínez A, López-Guillermo A, et al. Diffuse large B-cell lymphomas with plasmablastic differentiation represent a heterogeneous group of disease entities. Am J Surg Pathol 2004; 28 : 736-47.

22.- Carbone A, Gloghini A, Vaccher E, Cerri M, Gaidano G, Dalla-Favera R, et al. Kaposi's sarcoma-associated herpesvirus/human herpesvirus type 8-positive solid lymphomas: a tissue-based variant of primary effusion lymphoma. J Mol Diagn 2005; 7: 17-27.

23.- Castillo J J. Winer E S, Stachurski D, Pérez K, Jabbour M, Milani C, et al. Prognostic factors in chemotherapy-treated patients with HIV-associated plasmablastic lymphoma. Oncologist 2010; 15: 293-9.

24.- Little R F, Pittaluga S, Grant N, Steinberg S M, Kavlick M F, Mitsuya H, et al. Highly effective treatment of acquired immunodeficiency syndrome-related lymphoma with dose-adjusted EPOCH: impact of antiretroviral therapy suspension and tumor biology. Blood 2003; 101: 4653-9. 